\title{
Laser micro-engineering of functionalized cyclic olefin polymers for microfluidic applications
}

\author{
Ronán McCann*a,b,c, Komal Bagga ${ }^{\mathrm{a}, \mathrm{b}}$, Apryll Stalcup ${ }^{\mathrm{b}, \mathrm{d}}$, Mercedes Vázquez ${ }^{\mathrm{a}, \mathrm{b}, \mathrm{d}}$, Dermot Brabazon ${ }^{\mathrm{a}, \mathrm{b}, \mathrm{c}}$ \\ ${ }^{a}$ Advanced Processing Technology Research Centre, School of Mechanical Engineering, Dublin City \\ University, Ireland; ' Irish Separation Science Cluster, National Centre for Sensor Research, Dublin \\ City University, Ireland; ' National Centre for Plasma Science and Technology, Dublin City \\ University, Ireland; ${ }^{\mathrm{d}}$ School of Chemical Sciences, Dublin City University, Ireland.
}

\begin{abstract}
Direct-write laser processing has been demonstrated to be capable of both surface patterning of micro- and nanoscale structures on polymer surfaces without significant modification of the surface chemistry or optical transmission of the laser processed area. In this work, the creation of microchannels via direct-write laser processing of $188 \mu \mathrm{m}$ thickness cyclic olefin polymers is demonstrated, along with a route towards channel functionalization. Cyclic olefin polymers (COP) are an emerging class of polymers noted for their high chemical resistance, biocompatibility and higher optical transparency when compared to other common polymers. These properties make them excellent substrates for the fabrication of microfluidic devices. This paper presents the first investigation into infrared laser processing of COP using a $1064 \mathrm{~nm} \mathrm{Nd:YAG} \mathrm{laser.} \mathrm{Scanning} \mathrm{electron} \mathrm{microscopy} \mathrm{and} \mathrm{Raman} \mathrm{spectroscopy} \mathrm{were} \mathrm{utilized} \mathrm{to} \mathrm{investigate} \mathrm{the}$ morphology and composition of these laser textured surfaces.

A route for functionalization of these substrates for chemical and biological speciation and separation was examined using carbon nanoparticles. The nanoparticles were produced using pulsed laser ablation in liquid (PLAL) which has been reported as a fast and adaptable method for nanoparticle production. The nanoparticles produced were using transmission electron microscopy while the coating of substrates with these CNPs was examined using SEM. These results are discussed in the context of development of a new route for achieving surfaces optimized for microfluidicbased separations and speciation.
\end{abstract}

Keywords: Cyclic olefin polymer, pulsed laser ablation, carbon nanoparticles, functionalization, microfluidics, Nd:YAG,

\section{INTRODUCTION}

Cyclic olefin polymer (COP), and its co-polymer variant (COC), are promising classes of polymers noted for their exceptional properties such as high optical transparency from mid-UV to near-IR wavelengths, low cost, biocompatibility, strong chemical resistance to polar solvents, and high mechanical and dimensional stability when in contact with liquids ${ }^{1}$. To date, COPs have been demonstrated in a wide range of applications such as IR-waveguide coatings $^{2}$, IR lenses ${ }^{3}$ as well as lithographic photoresist ${ }^{4}$. Another area of application which takes advantage of COPs excellent properties is the field of microfluidics. Typically, these microfluidic platforms would be fabricated from glass or polymers such as poly(methyl methacrylate) (PMMA), polycarbonate (PC) or polydimethylsiloxane (PDMS). Though these polymeric materials have good mechanical and thermal properties, thus allowing for easy processing, they tend to lack the optical transparency in the UV range necessary for the optical analysis of aromatics, proteins and nucleic acids. COPs remarkable optical and mechanical properties make them extremely suitable replacements for fabrication of microfluidic devices.

Polymeric microfluidic device fabrication can be done via techniques such as xurography ${ }^{5}$, micromilling ${ }^{6}$, UVpolymerisation $^{7}$ and more recently, 3D printing ${ }^{8,9}$. These processes, while allowing for rapid prototyping and device optimization, are difficult or are not conventionally utilized for mass production. Laser processing however, allows for both rapid device creation and high-throughput. Though laser processing of other polymers for microfluidic device

*ronan.mccann5@mail.dcu.ie; phone +353 1700 7674; fax +353 1700 7148; www.dcu.ie/apt 
creation have been studied ${ }^{10,11}$, few studies to date have investigated the possibility of laser processing either COP or COC. Sabbert et al. studied ablation of COC using an ArF excimer laser ${ }^{12}$ and showed the ability of this system to engineer high precision, micron size square holes was shown. The Ablation depth per pulse for COC was found 3.4 times smaller than for PMMA, a property which could be exploited for the fabrication of microchannel structures. In another study by Suriano et al. explored the use of a femtosecond titanium sapphire laser (800 nm) for microchannel fabrication on COP, PMMA and polystyrene (PS) substrates. V-shaped microchannels on COP ranging in depth from 10 $\mu \mathrm{m}$ up to $80 \mu \mathrm{m}$ were created, with channel roughness comparable to those created through micromilling. No study has yet examined the use of $\mathrm{Nd}$ :YAG infrared lasers, which are widely used in industry for material processing ${ }^{13}$, for the processing of COPs.

Though COP based substrates offer excellent platforms for microfluidic devices, additional functionalization is required for use in chemical and biological speciation applications. Nanoparticle (NP) coatings offer the capability of providing novel and adaptable substrate functionalization. The use of Pulsed Laser Ablation in Liquid (PLAL), unlike other forms methods of NP production, allows for the synthesis of chemically highly pure NPs. Furthermore, similarly to laser processing, PLAL is a fast process allowing for rapid production and optimization of the produced NP suspensions. In this work, IR $1064 \mathrm{~nm}$ laser processing of $188 \mu \mathrm{m}$ thick COP using a $1064 \mathrm{~nm}$ laser as a potential route to create microfluidic devices was investigated. Additionally, the production of carbon nanoparticles through PLAL and their potential use for functionalization of laser produced microchannels was also examined with an aim to develop a route for rapid creation fabrication of chemical or biological speciation platforms.

\section{EXPERIMENTAL}

\section{1. $1064 \mathrm{~nm}$ laser processing system}

The laser system used (shown in Figure 1) consisted of a Q-switched diode-pumped solid state (DPSS) $1064 \mathrm{~nm}$ neodymium-doped yttrium aluminum garnet (Nd:YAG). The laser had a maximum average power output of $4 \mathrm{~W}$, a pulse width of $750 \mathrm{ps}$ and a pulse repetition frequency of $7.5 \mathrm{kHz}$. A 2-dimensional galvanometer (SS-12, Raylase) provided $\mathrm{x}$ - and y-positioning of the laser beam and a movable z-stage (M-404 4PD, Physik Instrumente) controlled the sample position in the laser focal plane.

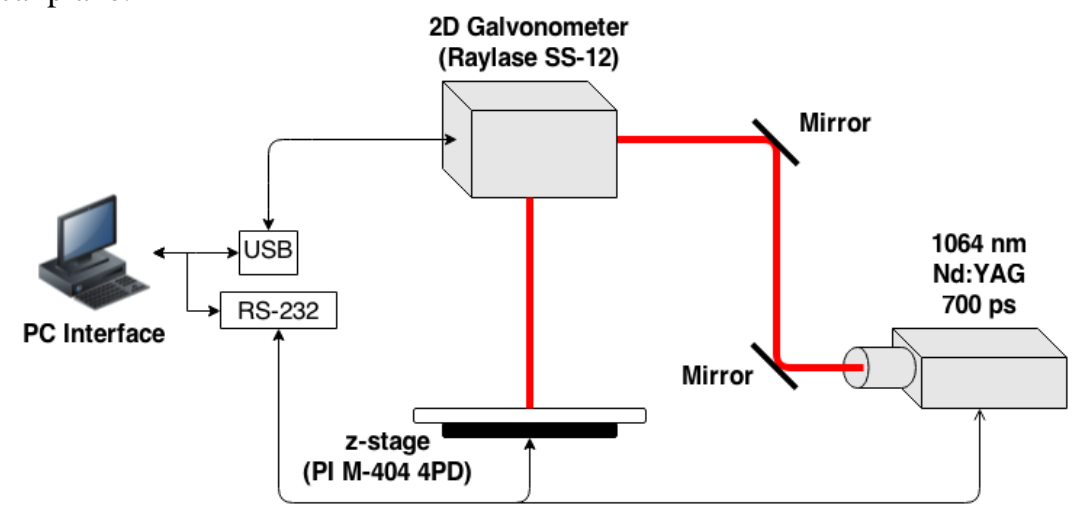

Figure 1. Schematic of the $1064 \mathrm{~nm} \mathrm{Nd:YAG} \mathrm{laser} \mathrm{processing} \mathrm{system} \mathrm{used} \mathrm{for} \mathrm{the} \mathrm{experiment.}$

\subsection{Laser texturing of substrates}

The cyclic olefin polymer substrates used had a thickness of $188 \mu \mathrm{m}$ (ZF14-188 ZeonorFilm®, Zeon Chemical, Japan) with a thickness of $188 \mu \mathrm{m}$. The polymer substrates were cut, cleaned, washed with isopropanol (>70\%) and rinsed with deionized water (DI water) and dried with compressed clean dry air to ensure the substrate was pristine prior to laser processing. The laser beam was focused to a spot diameter of $140 \mu \mathrm{m}$ and scanned unidirectionally across the sample at a speed of $1.2 \mathrm{~mm} / \mathrm{s}$ to form channels separated by $200 \mu \mathrm{m}$. For the whole duration of the processing, the sample was mechanically clamped. The substrates were processed in two phases. During the first phase, a high fluence pass was applied to ablate the substrate surface and form the microchannel. This was then followed by a number of low fluence passes to remove condensed melt and ablated particulates from the microchannel. The fluence was set at $0.51 \mathrm{~J} / \mathrm{cm}^{2}$ and $0.34 \mathrm{~J} / \mathrm{cm}^{2}$ for the high and low passes, respectively. 


\subsection{Synthesis of nanoparticles and substrate coating}

Synthesis of the carbon nanoparticles was conducted via PLAL using the $1064 \mathrm{~nm} \mathrm{Nd:YAG} \mathrm{as} \mathrm{described} \mathrm{previously} \mathrm{by}$ Bagga et al. ${ }^{14}$ using the same laser used to produce the substrates. A cylindrical graphite target $(>99.999 \%$, Sigma) with a diameter of $6 \mathrm{~mm}$ and height of $8 \mathrm{~mm}$ was placed on the bottom of a glass cuvette $(10 \times 10 \times 50 \mathrm{~mm})$. The cuvette was filled with $2 \mathrm{~mL}$ of DI water, corresponding to $1 \mathrm{~cm}$ of liquid above the surface of the target. The target was polished mechanically and washed with DI water to ensure it was pristine prior to ablation. The laser ablation was conducted using a laser fluence ranging from $0.02 \mathrm{~J} / \mathrm{cm}^{2}$ to $0.71 \mathrm{~J} / \mathrm{cm}^{2}$.

Substrate coating was conducted by first activating the surface of as-received COP substrates using $20 \mu \mathrm{L}$ of toluene applied using a micropipette for around 10 seconds. Though COP exhibits high chemical resistance to acids, bases and alcohols, it is easily attacked by non-polar solvents such as toluene. After activation, a CNP suspension was drop cast onto COP substrates with a micropipette. The CNP-coated substrates were then left in an oven at $36.5^{\circ} \mathrm{C}$ for 24 hours to completely evaporate the toluene and isopropanol present on the substrate surface. Finally, these COP substrates were rinsed three times using deionized water and dried with compressed clean dry air, which was repeated three times per substrate. The substrates were sealed in petri dishes at room temperature for storage until analysis.

\subsection{Characterization}

Characterization of both the nanoparticles and the polymer substrates was performed using a number of techniques. Scanning electron microscopy (SEM) was performed to examine the microchannel morphology and nanoparticle coating using a Zeiss EVO LS15 (Carl Zeiss AG, Germany) at an accelerating voltage of $15 \mathrm{kV}$ and a beam intensity of $15 \mathrm{pA}$. The substrates were gold coated using a ScanCost Six (Edwards, UK) for $80 \mathrm{~s}$ at a sputter current of $25 \mathrm{~mA}$, with a resultant coating of $34 \mathrm{~nm}$. The surface was imaged at a $52^{\circ}$ tilt with respect to the normal to the electron beam emission direction.

Micro-Raman spectroscopy was performed on the CNPs with a Jobin-Yvon Horiba LabRam® HR800 at $20 \mathrm{~mW}$ and 1 $\mu \mathrm{m}^{2}$ spot size (using Ar+ $488 \mathrm{~nm}$ air cooled laser and an accumulation time of $20 \mathrm{~s}$ ) in backscatter configuration with a resolution of approximately $1.1 \mathrm{~cm}^{-1}$. Transmission electron microscopy (TEM) was employed to examine the nanoparticle morphology using a FEI Titan instrument, operating at $300 \mathrm{kV}$. Samples were prepared by drop casting onto a carbon coated 300 mesh copper grid and left to evaporate at room temperature.

\section{RESULTS}

\subsection{Substrate characterization}

For single laser passes, SEM imaging showed the formation microchannels with deep V-shaped profiles however the presence of localized ablation holes were seen at the bottom of the microchannels. For multiple laser passes, the microchannels became deeper with surface roughness along the channel base being reduced. This suggests suggested that both further material removal and a localized melting or annealing took place during successive passes. Though the localized ablation holes were still present on the base of the microchannels after multiple passes, the relative size was less when compared with the channel depths. Depths for the microchannels ranged from $22 \mu \mathrm{m}$ for a single high fluence pass, up to $77 \mu \mathrm{m}$ which resulted from one high fluence pass, in combination with eight low fluence passes.

Raman spectroscopy was performed to study the chemical composition after laser processing. The base of the microchannel was examined for changes, while the measurements from the interchannel areas (which were not processed, were used as a reference, see Figure 3. No significant differences in location were seen between the channel and interchannel peak positions. 

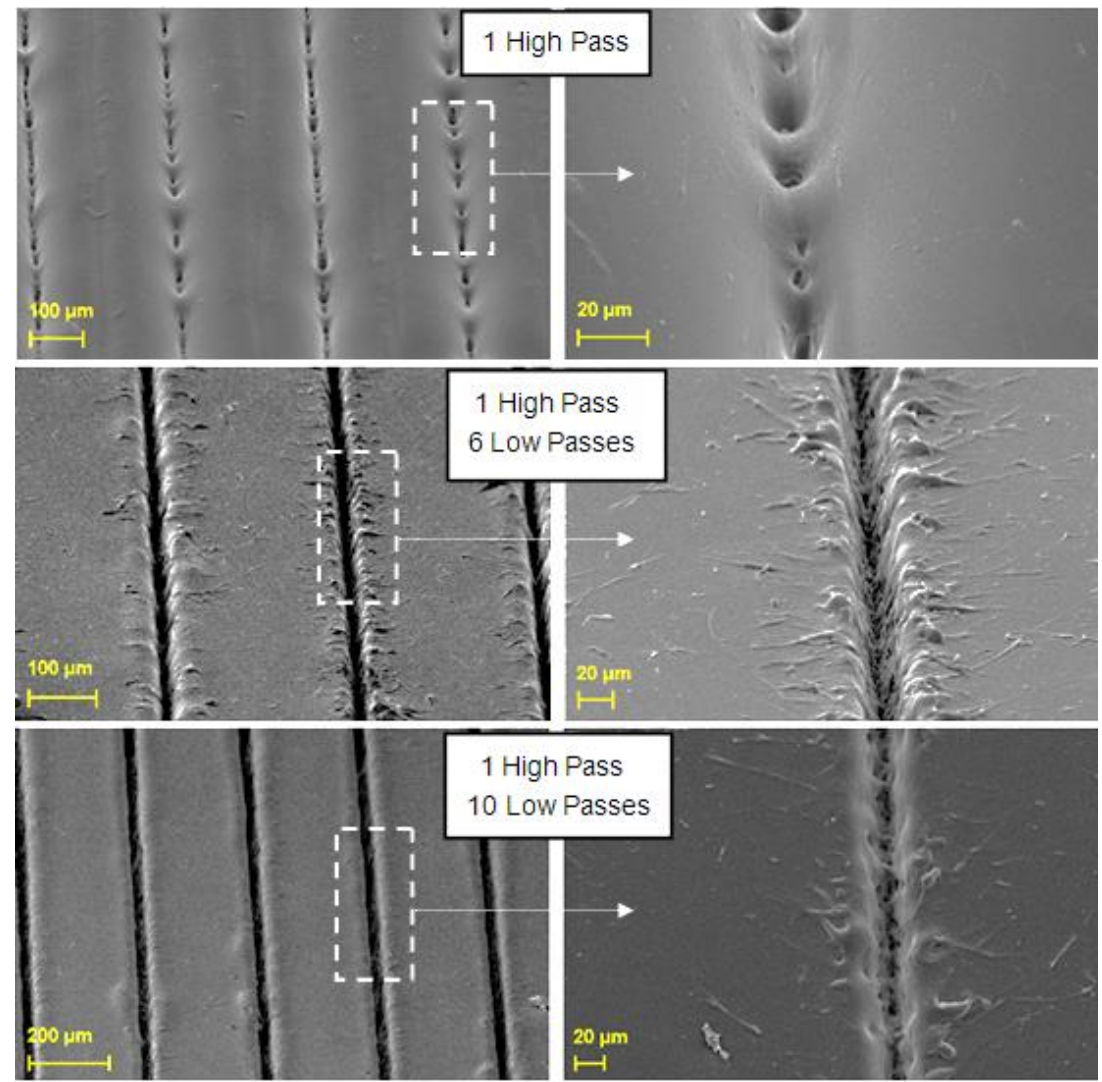

Figure 2. SEM images of the COP substrate after (top) 1 pass, (middle) 7 passes (1 high, 6 low), and (bottom) 11 passes (1 high, 10 low).
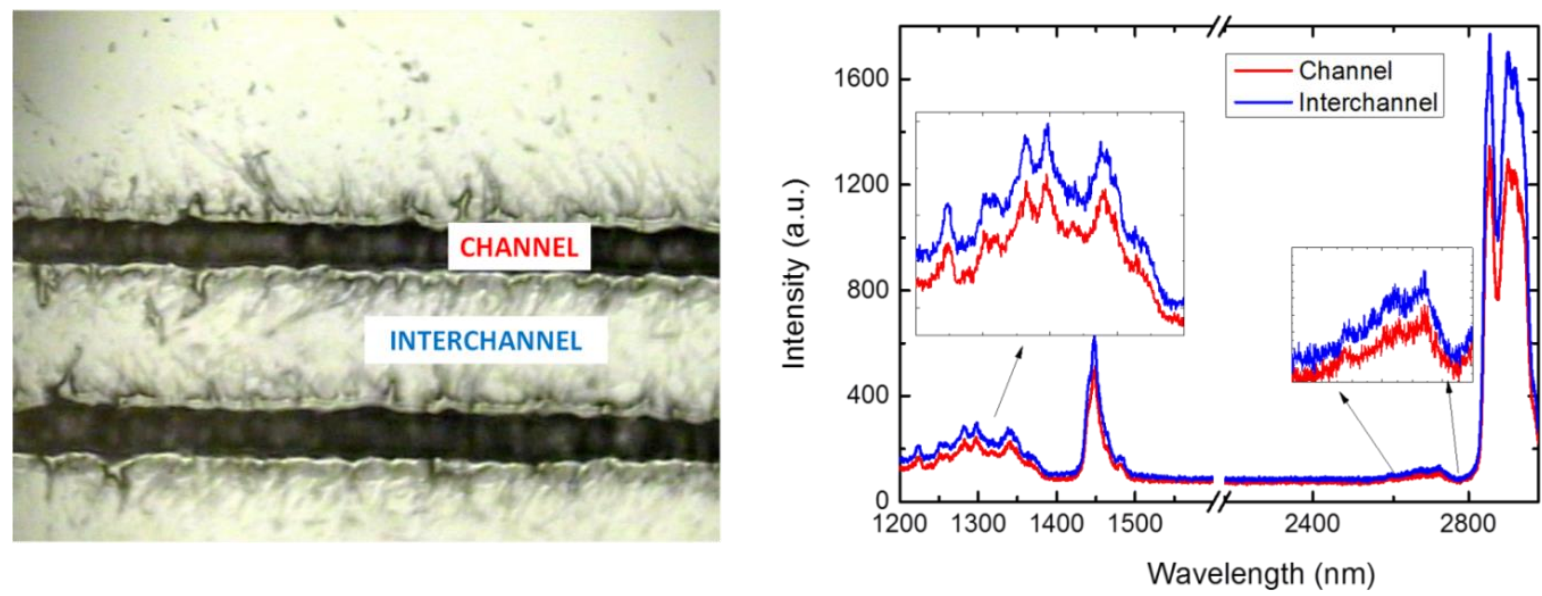

Figure 3: Micro-Raman spectroscopic analysis of the laser processed COP substrates. No significant compositional change was seen between the laser processed (channel) and reference (interchannel) areas.

\subsection{Nanoparticle morphology and substrate coating}

Figure 4 shows TEM images of the produced CNPs with a scale bar of (a) $100 \mathrm{~nm}$ and (b) $5 \mathrm{~nm}$. The nanoparticle size distribution was seen to be Gaussian in nature and ranged from $5 \mathrm{~nm}$ to $50 \mathrm{~nm}$ with a mean value of $18 \mathrm{~nm}$. The CNP nanoparticle colloid was drop cast onto the pristine COP and examined via SEM, see Figure 5. It can be seen that post 
coating, aligned CNP structures $(<200 \mathrm{~nm})$ formed. Larger CNPs clusters were also evident at discrete locations. Due to the cellular alignment of the smaller CNPs it is postulated that this CNP distribution originated mainly from the evaporation process. At lower concentrations, only the larger clusters formation was observed, demonstrating the need for optimization of the nanoparticle colloid concentration when performing substrate coating.

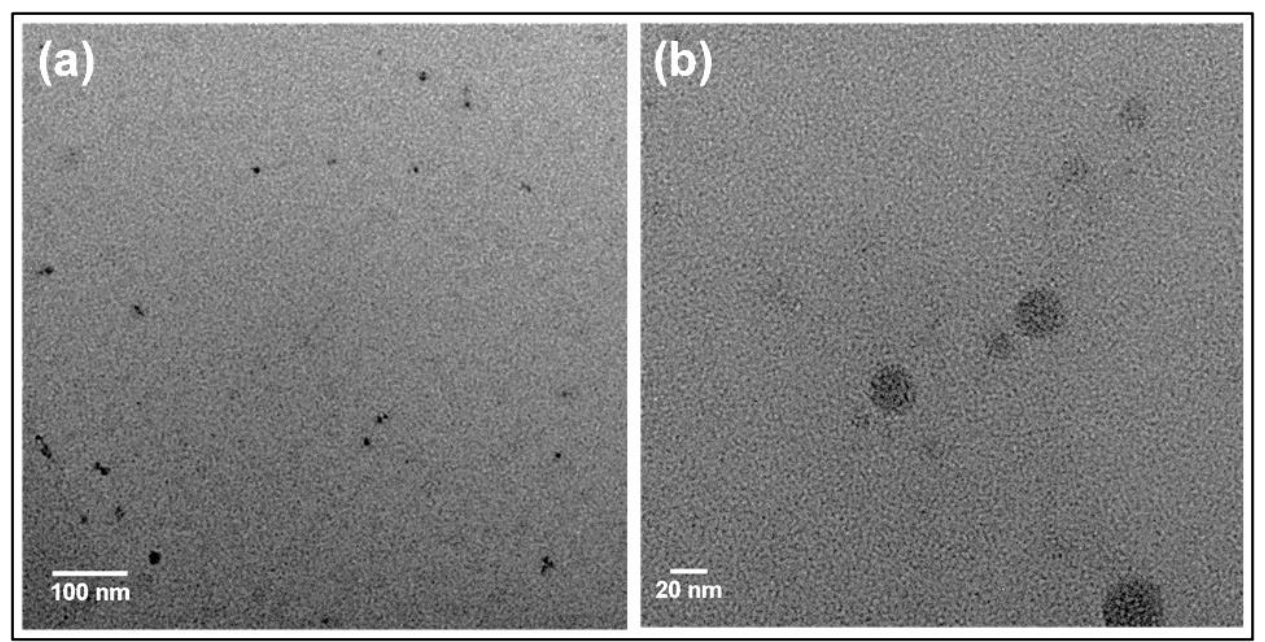

Figure 4. TEM analysis of CNPs produced via pulsed laser ablation in liquid in DI water at laser fluence of $0.40 \mathrm{~J} / \mathrm{cm}^{2}$.
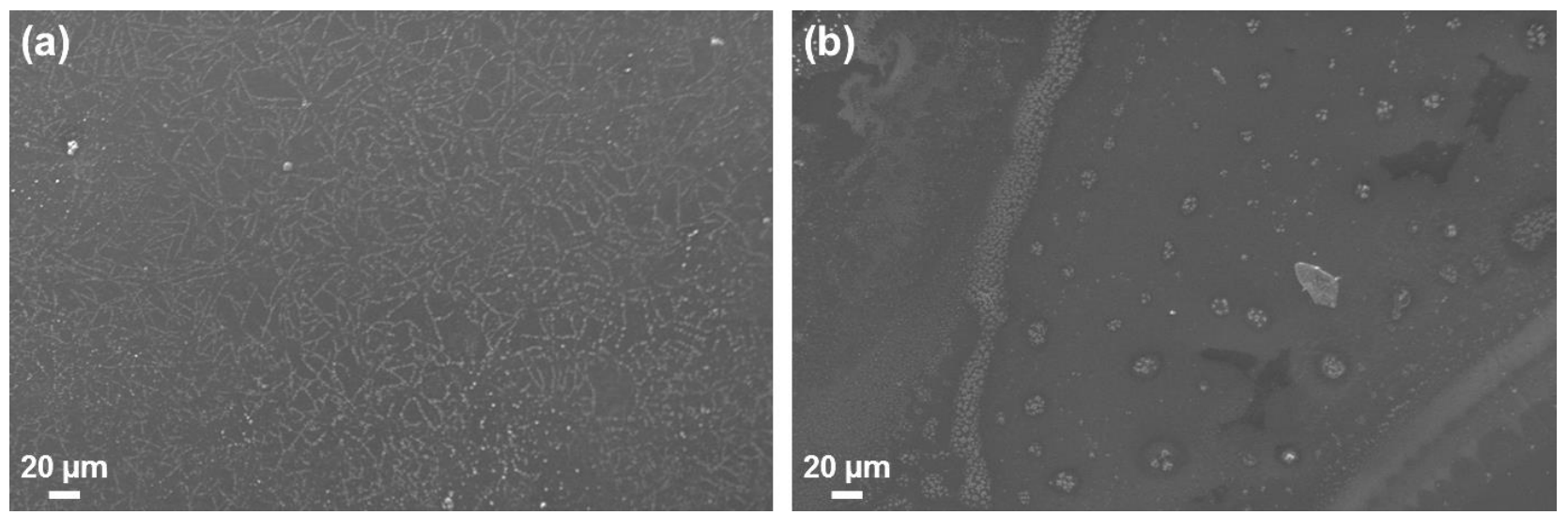

Figure 5. Carbon nanoparticles deposited on untextured COP. For higher CNP concentrations (a), the formation of structures was seen along with some larger nanoparticles clusters, while at lower concentrations (b) the formation of isolated clusters was apparent.

\section{DISCUSSION}

Laser processing was demonstrated to be capable of generating of micron sized channels on the surface of the thin polymer substrates. This capability, coupled with the speed and adaptability of laser processing, makes it an excellent route for the fabrication of microfluidic platforms. Raman spectroscopy confirmed the microchannels were not chemically altered by laser processing, thus the laser processed channels will presumably have the chemical resistance and biocompatibility of the unprocessed COP. This demonstrates that laser processing of COP is a viable route to generate substrates and devices suitable for microfluidics, where the chemistry of the surface in contact with the liquid plays a crucial role. Though the presence of localized ablation sites along the bottom surface of the microchannels was seen, further optimization of the laser processing parameters may allow for the creation of smoother and more uniform bottom surfaces of the microchannels.

Furthermore, COP's chemical resistance and biocompatibility make it extremely suitable for microfluidic applications once functionalized. To this end, carbon nanoparticles were produced via PLAL. The CNPs produced were seen to have 
a uniform Gaussian distribution, which demonstrates that PLAL is an excellent route for the fabrication of CNPs. Furthermore, the chemical purity of the CNPs produced via the PLAL method, as discussed in Section 1, could allow for a wide range of possible functionalization routes such as tosyl attachment ${ }^{15}$ which can be employed for the speciation of aromatic compounds. The drop cast coated substrates showed good coverage at higher CNP colloid concentrations, though lower concentrations only saw the presence of clusters. This demonstrates the need for optimization of the nanoparticle colloid concentration prior to drop casting. These nanoparticles, if coupled with the laser-processed COP substrates could allow for the fabrication of platforms with a wide range of applications in both chemical and biological speciation.

\section{CONCLUSIONS}

This article presents the first investigation into infrared laser processing of cyclic olefin polymers which was applied for the fabrication of microchannels or microfluidic applications. The novel use of an IR laser will allow for this fabrication technique to be used in both research and industrial environments. The ability to create microchannels ranging in depth from $22 \mu \mathrm{m}$ to $77 \mu \mathrm{m}$ was demonstrated using a $1064 \mathrm{~nm} \mathrm{Nd}$ :YAG laser. The chemical composition of the polymer postprocessing was not significantly changed, thereby leaving preserving COPs the high chemical resistance and biocompatibility which are necessary for many sensing and assay related applications. Functionalization by way of drop casting carbon nanoparticles produced via PLAL was also examined. PLAL was used as a fast and adaptable route to for CNP production, with the coated substrates showing good coverage of nanoparticles when produced with colloid of high concentration. In conclusion, both laser fabrication of the polymer substrates along with functionalization using CNPs provides a route to rapidly create functional surfaces for chemical and biological speciation and separation.

\section{REFERENCES}

[1] Nunes, P. S., Ohlsson, P. D., Ordeig, O.., Kutter, J. P., "Cyclic olefin polymers: emerging materials for lab-on-achip applications," Microfluid. Nanofluidics 9(2-3), 145-161 (2010).

[2] Shi, Y.-W., Wang, Y., Abe, Y., Matsuura, Y., Miyagi, M., Uyama, H., Taniwaki, M.., Sato, S., "Fabrication of cyclic olefin polymer (COP)-coated silver hollow glass waveguides for the infrared," Proc. SPIE - Int. Soc. Opt. Eng. 3262, M. S. Bogner, S. T. Charles, W. S. Grundfest, J. A. Harrington, A. Katzir, L. S. Lome, M. W. Vannier, and R. Von Hanwehr, Eds., 96-102 (1998).

[3] Ji, J., Fan, W., Kong, D.., Wang, L., "Design, fabrication and characteristics of cyclic olefin copolymers lens for terahertz application," Hongwai yu Jiguang Gongcheng/Infrared Laser Eng. 42(5), 1212-1217 (2013).

[4] Allen, R. D., Opitz, J., Wallow, T. I., Di Pietro, R. A., Hofer, D. C., Jayaraman, S., Hullihan, K. A., Rhodes, L. F., Goodall, B. L., et al., "Design of an etch-resistant cyclic olefin photoresist," Proc. SPIE - Int. Soc. Opt. Eng. 3333, W. Conley, Ed., 463-471 (1998).

[5] Azouz, A. Ben., Murphy, S., Karazi, S., Vázquez, M.., Brabazon, D., "Fast Fabrication Process of Microfluidic Devices Based on Cyclic Olefin Copolymer," Mater. Manuf. Process. 29(2), 93-99 (2014).

[6] Chen, P.-C., Pan, C.-W., Lee, W.-C.., Li, K.-M., "An experimental study of micromilling parameters to manufacture microchannels on a PMMA substrate,” Int. J. Adv. Manuf. Technol. 71(9-12), 1623-1630 (2014).

[7] Turri, S., Levi, M., Emilitri, E., Suriano, R.., Bongiovanni, R., "Direct Photopolymerisation of PEG-Methacrylate Oligomers for an Easy Prototyping of Microfluidic Structures," Macromol. Chem. Phys. 211(8), 879-887 (2010).

[8] Zhang, X., Jiang, X. .., Sun, C., "Micro-stereolithography of polymeric and ceramic microstructures," Sensors Actuators A Phys. 77(2), 149-156 (1999).

[9] Shallan, A. I., Smejkal, P., Corban, M., Guijt, R. M.., Breadmore, M. C., "Cost-effective three-dimensional printing of visibly transparent microchips within minutes.," Anal. Chem. 86(6), 3124-3130, American Chemical Society (2014).

[10] Cheng, J.-Y., Wei, C.-W., Hsu, K.-H.., Young, T.-H., "Direct-write laser micromachining and universal surface modification of PMMA for device development," Sensors Actuators B Chem. 99(1), 186-196 (2004).

[11] Suriano, R., Kuznetsov, A., Eaton, S. M., Kiyan, R., Cerullo, G., Osellame, R., Chichkov, B. N., Levi, M.., Turri, S., "Femtosecond laser ablation of polymeric substrates for the fabrication of microfluidic channels," Appl. Surf. Sci. 257(14), 6243-6250 (2011).

[12] Sabbert, D., Landsiedel, J., Bauer, H.-D.., Ehrfeld, W., “ArF-excimer laser ablation experiments on Cycloolefin Copolymer (COC),” Appl. Surf. Sci. 150(1-4), 185-189 (1999). 
[13] Prakash, S., Acherjee, B., Kuar, A. S.., Mitra, S., “An experimental investigation on Nd:YAG laser microchanneling on polymethyl methacrylate submerged in water," Proc. Inst. Mech. Eng. Part B J. Eng. Manuf. 227(4), 508-519, SAGE Publications (2013).

[14] Bagga, K., McCann, R., Wang, M., Stalcup, A., Vázquez, M.., Brabazon, D., "Laser assisted synthesis of carbon nanoparticles with controlled viscosities for printing applications," J. Colloid Interface Sci. (2014).

[15] Vidal, L., Chisvert, A., Canals, A., Psillakis, E., Lapkin, A., Acosta, F., Edler, K. J., Holdaway, J. A.., Marken, F., "Chemically surface-modified carbon nanoparticle carrier for phenolic pollutants: Extraction and electrochemical determination of benzophenone-3 and triclosan.," Anal. Chim. Acta 616(1), 28-35 (2008).

\section{ACKNOWLEDGMENTS}

This publication has emanated from research conducted with the financial support of Science Foundation Ireland (SFI) under Grant Number 12/IA/1576. 